\title{
Dense matter in strong magnetic fields without nucleons
}

\author{
Tomáš Brauner \\ Department of Mathematics and Physics \\ University of Stavanger, Norway \\ E-mail: tomas.brauner@uis.no
}

The ground state of QCD in sufficiently strong magnetic fields and at nonzero baryon chemical potential is a topological crystal made of neutral pions: the Chiral Soliton Lattice (CSL). Due to its topological nature, it carries nonzero baryon number density that can reach values relevant for the cores of neutron stars. The spectrum of excitations above the CSL ground state contains a soft, nonrelativistic mode that gives an anomalous contribution to pressure, scaling with temperature and magnetic field as $T^{5 / 2} B^{3 / 2}$. In stronger but still achievable magnetic fields, the neutral pion CSL background may catalyze Bose-Einstein condensation of charged pions.

XIII Quark Confinement and the Hadron Spectrum - Confinement2018

31 July - 6 August 2018

Maynooth University, Ireland 


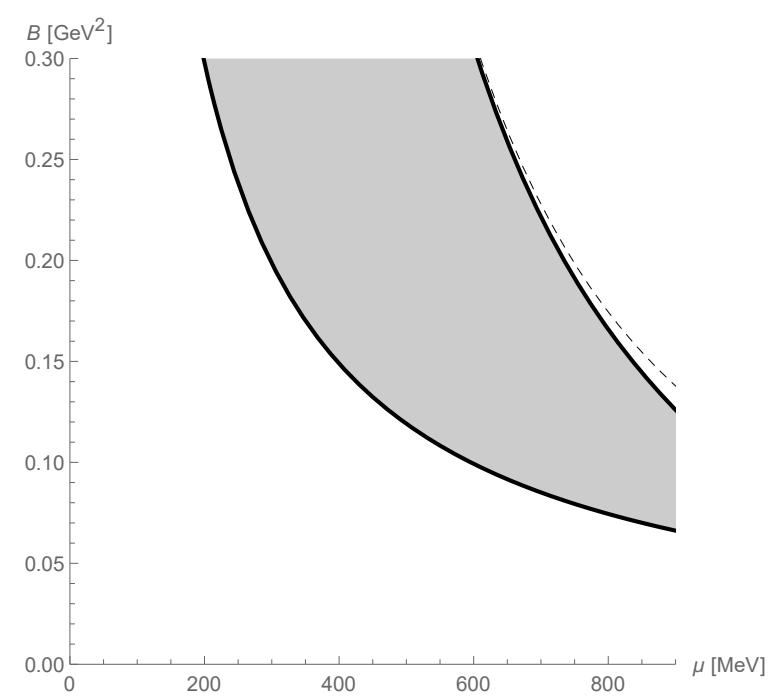

Figure 1: Phase diagram of QCD with two light quark flavors as a function of baryon number chemical potential and magnetic field at zero temperature. The lower solid line indicates a phase transition from the usual chiral-symmetry-breaking vacuum to the CSL phase (shaded area). Above the upper solid line, the CSL state becomes unstable with respect to Bose-Einstein condensation of charged pions. The dashed line indicates the same instability in the chiral limit. The numerical values were obtained using $f_{\pi}=92 \mathrm{MeV}$ and $m_{\pi}=140 \mathrm{MeV}$ as input.

\section{Introduction and the main result}

The phase structure of quantum chromodynamics (QCD) in strong external magnetic fields has been subject to intensive scrutiny in the past decade or so [1,2]. Apart from the intrinsic interest of the subject, this is largely motivated by the experimental studies of relativistic heavy ion collisions, and by the physics of neutron stars.

In this contribution, I report on the results obtained in collaboration with Naoki Yamamoto [3] and in the follow-up work with Saurabh Kadam [4]. Our main result is displayed in figure 1. This is the phase diagram of QCD as a function of baryon number chemical potential $\mu$ and external magnetic field $\boldsymbol{B}$ at zero temperature. Note that a nontrivial phase structure exists even for chemical potentials $\mu<m_{\mathrm{N}}$, where $m_{\mathrm{N}}$ is the nucleon mass. This is a consequence of the interaction of quarks with the external magnetic field. The shaded area indicates a new phase of quark matter, dubbed the Chiral Soliton Lattice (CSL). This phase carries a spatially inhomogeneous condensate of neutral pions. Due to its topological nature, the condensate carries nonzero baryon number, and as such represents "nuclear matter" in a thermodynamic regime where nucleons cannot exist.

This main result was obtained at strictly zero temperature, although as I will argue later, the nonuniform long-range order of the CSL phase is expected to persist up to certain nonzero critical temperature. Also, the CSL state requires for its existence strong magnetic fields of the order of $10^{19} \mathrm{G}$. Let me, however, stress that this is not a result of a model calculation. On the contrary, it is a model-independent prediction that follows directly from the low-energy effective field theory (EFT) of QCD. Thus, figure 1 is, in fact, the phase diagram of QCD itself, obtained in an approximation defined by the truncation of the derivative expansion of the low-energy EFT. 


\section{Low-energy effective theory of QCD in strong magnetic fields}

The low-energy EFT of QCD with two degenerate quark flavors is defined by the Lagrangian

$$
\mathscr{L}=\frac{f_{\pi}^{2}}{4}\left[\operatorname{Tr}\left(D_{\mu} \Sigma^{\dagger} D^{\mu} \Sigma\right)+m_{\pi}^{2} \operatorname{Tr}\left(\Sigma+\Sigma^{\dagger}\right)\right]+\mathscr{L}_{\mathrm{WZ}}
$$

Here $\Sigma$ is a unimodular unitary $2 \times 2$ matrix field that encodes the low-energy degrees of freedom: the pion triplet. Furthermore, $f_{\pi}$ is the pion decay constant and $m_{\pi}$ the pion mass. The coupling to an external electromagnetic field is defined via the covariant derivative, $D_{\mu} \Sigma \equiv \partial_{\mu} \Sigma-\mathrm{i} A_{\mu}[Q, \Sigma]$, where $Q \equiv \operatorname{diag}\left(\frac{2}{3},-\frac{1}{3}\right)$ is the electric charge operator in the quark flavor space and $A_{\mu}$ is the electromagnetic gauge potential.

The first term in eq. (2.1) corresponds to the leading order of the derivative expansion of the EFT. To this, the contribution of the chiral anomaly, $\mathscr{L}_{\mathrm{WZ}}$, is added, which will turn out to play a key role for the existence of the CSL phase. The precise dependence of the full anomalous (WessZumino) Lagrangian on the matrix field $\Sigma$ is immaterial; see for instance ref. [5] for details. What matters is that it generates a coupling of neutral pions to the electromagnetic field $A_{\mu}$ as well as to the baryon number chemical potential $\mu$.

In a constant background magnetic field $\boldsymbol{B}$, the stationary states of charged pions will be organized into Landau levels. The energy gap of the charged pions will accordingly read $\sqrt{m_{\pi}^{2}+|\boldsymbol{B}|}$. As a consequence, the charged pions are expected to decouple from the EFT for fields $B \equiv|\boldsymbol{B}| \gtrsim m_{\pi}^{2}$. In sufficiently strong magnetic fields, the low-energy physics of QCD will be dominated by neutral pions. The corresponding low-energy effective Lagrangian is obtained from eq. (2.1) by setting $\Sigma \rightarrow e^{\mathrm{i} \tau_{3} \phi}$, where $\tau_{3}$ is the third Pauli matrix and $\phi$ is a dimensionless neutral pion field. A Legendre transform then leads to the Hamiltonian

$$
\mathscr{H}=\frac{f_{\pi}^{2}}{2}(\nabla \phi)^{2}+m_{\pi}^{2} f_{\pi}^{2}(1-\cos \phi)-\frac{\mu}{4 \pi^{2}} \boldsymbol{B} \cdot \nabla \phi,
$$

where a constant has been added to ensure that the energy of the trivial (chiral-symmetry-breaking) vacuum is zero. The last term in eq. (2.2) is all that is left of the anomalous Lagrangian $\mathscr{L}_{\text {WZ }}$ in presence of a constant magnetic field when only the neutral pion degree of freedom is retained.

\section{Chiral soliton lattice}

A quick glance at the Hamiltonian (2.2) is enough to convince oneself that the chiral anomaly in presence of magnetic field and baryon chemical potential favors neutral pion field configurations, spatially modulated in the direction of the magnetic field [5], which shall without loss of generality be denoted as $z$. Gradients of $\phi$ in the transverse directions only enter the Hamiltonian through the first, manifestly positive-definite term. Hence the ground state of the Hamiltonian can only depend on the $z$-coordinate.

The derivation of the ground state is most conveniently divided into two steps. First, note that the anomalous contribution to the Hamiltonian is a surface term thanks to the Bianchi identity $\boldsymbol{\nabla} \cdot \boldsymbol{B}=0$. As such, it does not affect the equation of motion for $\phi$. To find the ground state, one can therefore first analyze the solutions of the equation of motion, and then pick the solution that has the lowest energy. The point of this at first complicated-looking procedure is that in one 

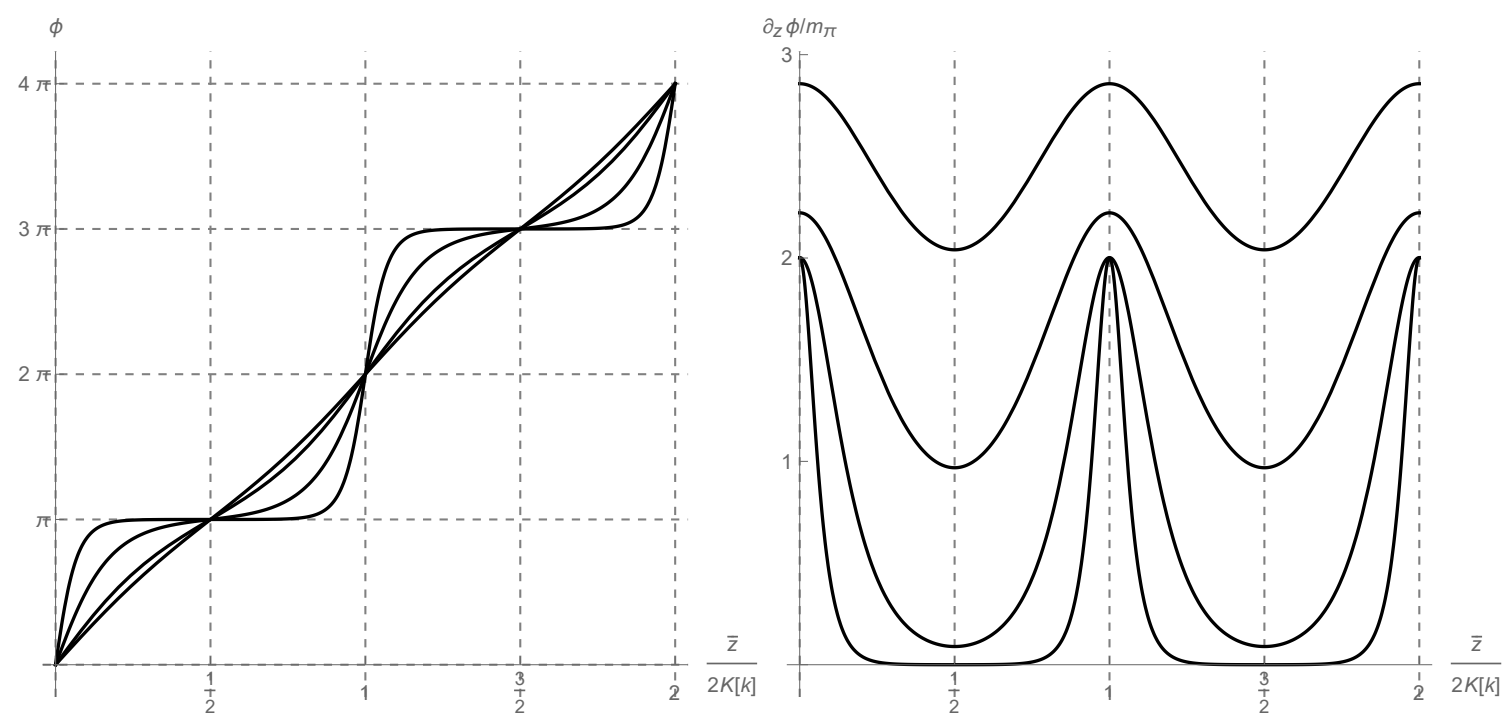

Figure 2: Left panel: solution to the classical equation of motion. For $k \rightarrow 0$, the solution is nearly linear with a small periodic oscillation. For $k \rightarrow 1$, the solution is step-like. The displayed curves correspond to $k=0.7,0.9,0.999,0.999999999$. Right panel: the gradient of the solution. The displayed curves correspond to the same values of $k$ as in the left panel, with $k$ increasing from the top downwards. The coordinate shown on the horizontal axes has been rescaled to allow comparison of the different solutions. For convenience, the angle $\phi$ has been shifted by $\pi$, which corresponds to replacing the cosine with a sine in eq. (3.1).

dimension, the Hamiltonian (2.2) without the last term is well-understood: it is namely identical to the Hamiltonian of the sine-Gordon model. Solving the corresponding equation of motion is then equivalent to understanding the dynamics of a simple pendulum, where the $z$-coordinate is mapped on the suitably rescaled time variable and the pion mass squared $m_{\pi}^{2}$ on the gravitational acceleration $g$. Using this analogy, it is easy to verify that, up to a shift of the coordinate, the general solution of the equation of motion can be written in terms of the Jacobi elliptic functions,

$$
\cos \frac{\phi(\bar{z})}{2}=\operatorname{sn}(\bar{z}, k)
$$

where $\bar{z} \equiv z m_{\pi} / k$ is a dimensionless coordinate and $k$ is the so-called elliptic modulus [6].

This is a one-parameter family of solutions and the elliptic modulus can be thought of as measuring the total mechanical energy of the pendulum. Solutions for which the pendulum undergoes periodic oscillations are not interesting, since for such solutions the anomalous contribution to the Hamiltonian (2.2) gives zero when integrated over one period, and the ground state is obviously realized by constant $\phi$. On the contrary, solutions for which the pendulum has sufficient energy to pass through its highest point are topologically nontrivial in that the angle $\phi$ accumulates nonzero winding number. Consequently, the contribution of the magnetic field to the energy as defined by the Hamiltonian (2.2) will be nontrivial. Such solutions are characterized by an elliptic modulus in the range $0 \leq k \leq 1$, and are illustrated in the left panel of figure 2 .

The form of the Hamiltonian (2.2) makes it clear that a spatially modulated solution of the 


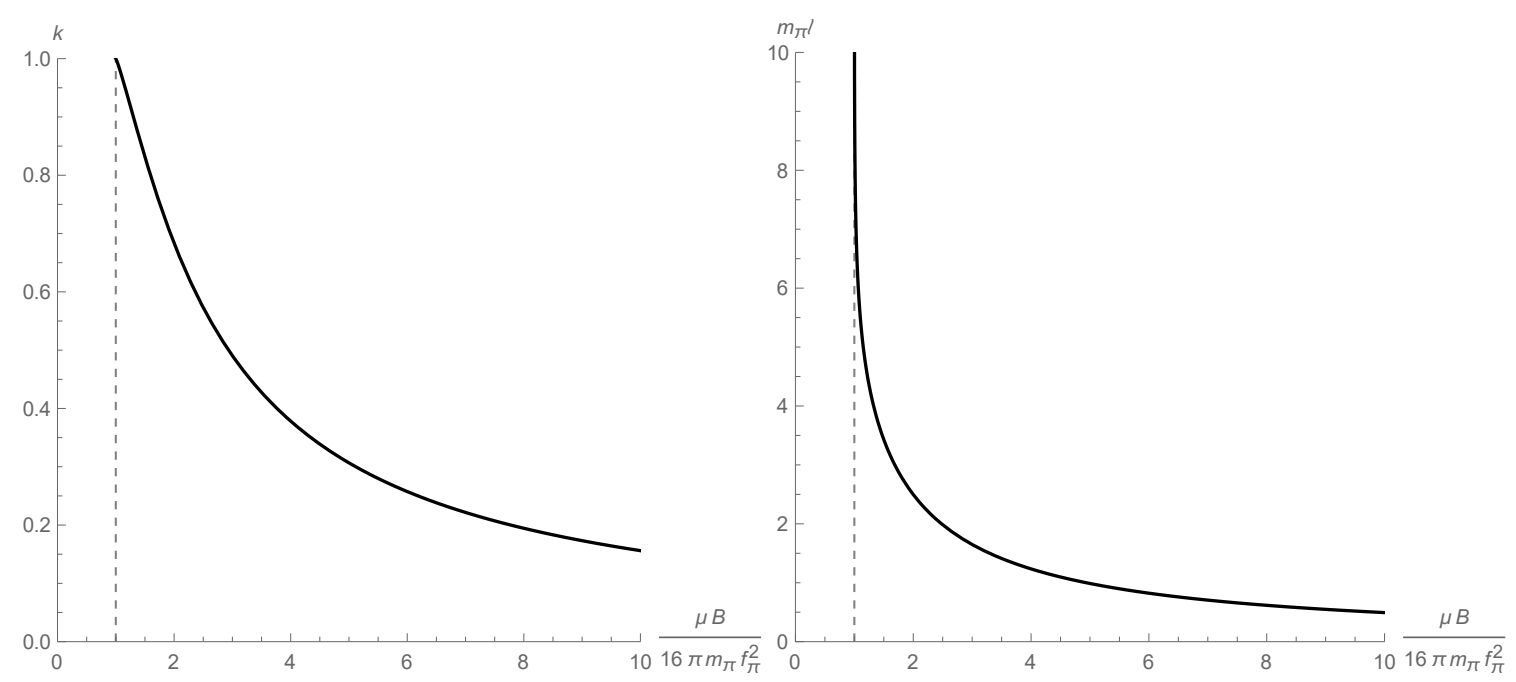

Figure 3: Left panel: optimal value of the elliptic modulus $k$ as a function of a dimensionless combination of the chemical potential $\mu$ and the magnetic field $B$. Right panel: the dimensionless lattice spacing of the CSL solution as a function of the same combination of $\mu$ and $B$. The critical magnetic field for the onset of the CSL phase corresponds to $\mu B=16 \pi m_{\pi} f_{\pi}^{2}$. At this point, a single domain wall first appears, corresponding to $k=1$ and a diverging lattice spacing.

equation of motion carries nonzero baryon number density,

$$
n_{\mathrm{B}}=\frac{1}{4 \pi^{2}} \boldsymbol{B} \cdot \boldsymbol{\nabla} \phi .
$$

Up to a normalization factor, this is shown in the right panel of figure 2. Although the low-energy EFT (2.1) only contains mesonic degrees of freedom, baryon number is generated as a topological charge, in a manner somewhat similar to the old Skyrme model of baryons. Figure 2 demonstrates that the class of field configurations described here has the structure of a topological lattice. In the limit $k \rightarrow 1$, it can be regarded as a lattice of well-separated domain walls [5]. Since nonzero $\phi$ implies a nonzero condensate of neutral pions, the state at the same time breaks the chiral symmetry, hence the name Chiral Soliton Lattice (CSL).

In order to determine which of the found solutions corresponds to the actual ground state of the Hamiltonian (2.2) for given chemical potential $\mu$ and magnetic field $\boldsymbol{B}$, one needs to evaluate the spatially averaged energy density and minimize it with respect to the elliptic modulus $k$, which until now has been a free parameter. This leads to the condition

$$
\frac{E(k)}{k}=\frac{\mu B}{16 \pi m_{\pi} f_{\pi}^{2}},
$$

where $E(k)$ is the complete elliptic integral of the second kind. A numerical solution for the elliptic modulus $k$ as a function of $\mu$ and $B$ is displayed in the left panel of figure 3. It indicates that the CSL state is energetically favored compared to the QCD vacuum for fields above certain critical value $B_{\mathrm{CSL}}$, given by

$$
\mu B_{\mathrm{CSL}}=16 \pi m_{\pi} f_{\pi}^{2} .
$$




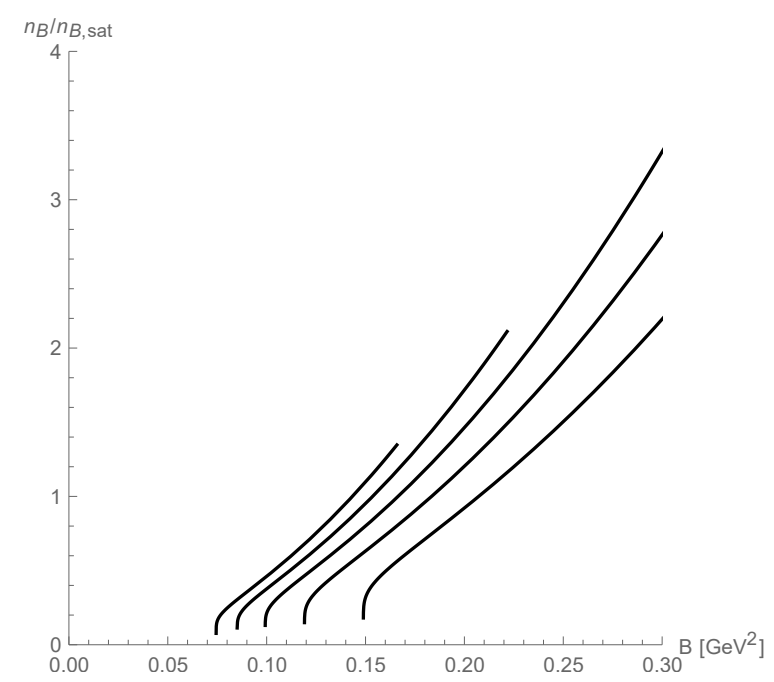

Figure 4: Spatially averaged baryon number density of the CSL solution in units of the nuclear saturation density as a function of the magnetic field. The five displayed curves correspond, from right to left, to $\mu=$ 400,500,600,700,800 MeV. The numerical values were obtained using $f_{\pi}=92 \mathrm{MeV}$ and $m_{\pi}=140 \mathrm{MeV}$ as input.

This equation defines the phase transition to the CSL phase, shown in figure 1. The lattice spacing of the CSL solution is given by the period of the Jacobi elliptic functions, and equals

$$
\ell=\frac{2 k K(k)}{m_{\pi}}
$$

where $K(k)$ is the complete elliptic integral of the first kind. This is displayed in the right panel of figure 3. Just above the critical magnetic field $B_{\mathrm{CSL}}$, the CSL solution takes the form of a lattice of thin, well-separated domain walls. As the magnetic field increases, however, the width of the domain walls rapidly increases and their separation decreases. At fields just a few times the critical field $B_{\mathrm{CSL}}$, the CSL profile can be very accurately approximated by a monochromatic standing wave [4].

Finally, let us have a look at the spatially averaged baryon number density, associated with the CSL state. This is displayed in figure 4 as a function of magnetic field for several different values of the chemical potential. The unit used on the vertical axis is the nuclear saturation density, $n_{\mathrm{B}, \text { sat }} \approx 0.16 \mathrm{fm}^{-3}$. One can see that densities of several times the saturation density can easily be achieved, which hints at possible relevance of the CSL phase for the physics of neutron stars.

\section{Excitation spectrum}

The spectrum of excitations above the CSL ground state can be studied either by expanding the Lagrangian to second order in small deviations of the fields from their vacuum expectation values, or equivalently by looking for solutions of the linearized equation of motion. The latter is particularly suitable for the case of the electrically neutral excitation, which has the quantum numbers of the neutral pion. 


\subsection{Neutral pion sector}

Keeping the notation $\phi$ for the CSL ground state and denoting the fluctuation of the phase as $\pi$, the linearized equation of motion follows directly from the Hamiltonian (2.2),

$$
\left(\square+m_{\pi}^{2} \cos \phi\right) \pi=0 .
$$

The $z$-dependence of the ground state allows one to Fourier transform in all spacetime coordinates but $z$ itself. Using the stationary state equation (3.1), the linearized equation of motion can then be cast as an eigenvalue problem for a certain differential operator acting on functions of $z$,

$$
\left[-\partial_{\bar{z}}^{2}+2 k^{2} \operatorname{sn}^{2}(\bar{z}, k)\right] \pi=\frac{k^{2}}{m_{\pi}^{2}}\left[m_{\pi}^{2}+\omega^{2}-\left(p_{x}^{2}+p_{y}^{2}\right)\right] \pi,
$$

where $\omega$ and $p_{x, y}$ are the frequency and momentum in the transverse directions, respectively. The left-hand side of eq. (4.2) is the so-called Lamé operator with index $n=1$. The wave functions of its eigenstates are well-known and can be expressed in terms of elliptic functions. The spectrum can, however, be found directly using algebraic methods [7, 8]. Given the periodic nature of the CSL ground state, the spectrum has a band structure. There are two energy bands, defined by the following algebraic conditions on the eigenvalues $\lambda$ of the Lamé operator,

$$
k^{2} \leq \lambda \leq 1, \quad 1+k^{2} \leq \lambda .
$$

This makes it clear that in terms of the frequency $\omega$, the lower, "valence" band is gapless in accord with the fact that the CSL state spontaneously breaks spatial translations in the $z$-direction. The phase velocity of the ensuing Nambu-Goldstone mode is given by

$$
c_{\mathrm{ph}}=\sqrt{1-k^{2}} \frac{K(k)}{E(k)},
$$

where $k$ is given implicitly by eq. (3.3). The dependence of the phase velocity on the magnetic field is shown numerically in the left panel of figure 5.

\subsection{Charged pion sector}

In order to understand the effect of the CSL background on the charged pions, one has to reintroduce them to the EFT, that is, return to the full Lagrangian (2.1). To study the fluctuations of the ground state, it is convenient to replace the matrix variable therein, $\Sigma \rightarrow e^{\mathrm{i} \tau_{3} \phi} U$, where $U$ is another unitary unimodular matrix, whose expectation value is the unit matrix, and it can thus be parameterized in terms of small field fluctuations as usual. Since the charged pions couple directly to the electromagnetic vector potential, it is next necessary to choose a gauge, which can be done by setting $\boldsymbol{A}=(0, B x, 0)$. The linearized equation of motion for the charged pion field $\pi^{+}$, already Fourier transformed in time and the $y$-coordinate, then reads

$$
\omega^{2} \pi^{+}=\left[-\partial_{x}^{2}+B^{2}\left(x-\frac{p_{y}}{B}\right)^{2}\right] \pi^{+}+\left(-\partial_{z}^{2}+2 \mathrm{i} \phi^{\prime} \partial_{z}+m_{\pi}^{2} e^{\mathrm{i} \phi}\right) \pi^{+},
$$

where the prime denotes a derivative with respect to $z$. In this equation, the $x$ - and $z$-coordinates are separated, which allows one to solve for the $x$-dependence in terms of Landau levels labeled by 

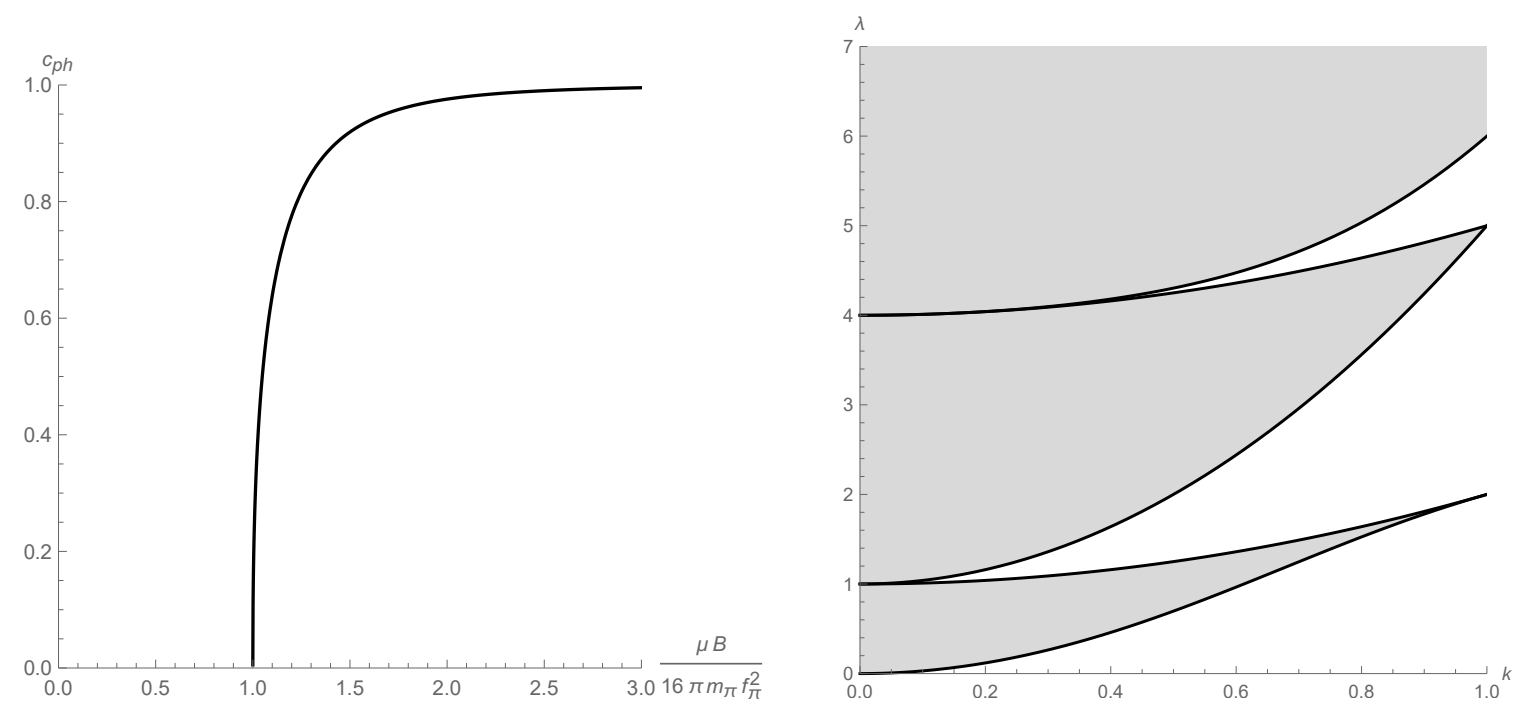

Figure 5: Selected properties of the spectrum of excitations above the CSL ground state. Left panel: phase velocity of the gapless neutral pion excitation as a function of magnetic field in units of the speed of light. Right panel: spectrum of eigenvalues of the $n=2$ Lamé operator, given by the left-hand side of eq. (4.6). The three displayed bands are defined by the inequalities in eq. (4.7).

a non-negative integer $N$. Upon a further redefinition $\pi^{+} \equiv e^{\mathrm{i} \phi} \tilde{\pi}^{+}$and upon using the CSL state condition (3.1), the equation of motion becomes

$$
\left[-\partial_{\bar{z}}^{2}+6 k^{2} \operatorname{sn}^{2}(\bar{z}, k)\right] \tilde{\pi}^{+}=\frac{k^{2}}{m_{\pi}^{2}}\left[\omega^{2}-(2 N+1) B\right] \tilde{\pi}^{+}+\left(k^{2}+4\right) \tilde{\pi}^{+} .
$$

The left-hand side of this equation is the Lamé operator with index $n=2$. The spectrum of its eigenvalues $\lambda$ consists of three bands as shown in the right panel of figure 5 , defined in turn by the algebraic conditions $[7,8]$

$2\left(1+k^{2}-\sqrt{1-k^{2}+k^{4}}\right) \leq \lambda \leq 1+k^{2}, \quad 1+4 k^{2} \leq \lambda \leq 4+k^{2}, \quad 2\left(1+k^{2}+\sqrt{1-k^{2}+k^{4}}\right) \leq \lambda$.

From eq. (4.6) it then follows that the bottom of the lowest energy band at the lowest Landau level is located at

$$
\min \omega_{N=0}^{2}=B-\frac{m_{\pi}^{2}}{k^{2}}\left(2-k^{2}+2 \sqrt{1-k^{2}+k^{4}}\right),
$$

where $k$ is given implicitly by eq. (3.3). In sufficiently strong magnetic fields, the lowest allowed energy of charged pions thus drops to zero in spite of the Landau level quantization of the spectrum. This is shown by the upper solid line in figure 1. For magnetic fields above this threshold, the CSL state is unstable and a new ground state, which includes a condensate of charged pions, emerges. The detailed structure of this ground state remains to be clarified though. In the chiral limit, it is straightforward to find the threshold for condensation of charged pions analytically. The result,

$$
B_{\mathrm{BEC}}=\frac{16 \pi^{4} f_{\pi}^{4}}{\mu^{2}}
$$

is indicated by the dashed line in figure 1 . 


\section{Coupling to dynamical electromagnetic field}

Until now, the external magnetic field was treated as a fixed background. This is most appropriate for lattice Monte Carlo simulations of the phase structure of QCD in strong magnetic fields. In reality, electromagnetic fields are dynamical though. It is then natural to ask how the anomalous coupling of the neutral pion to the electromagnetic field affects the propagation of photons in presence of the CSL background. To that end, we return to the EFT Lagrangian (2.1), discard as before the charged pions, but add the Lagrangian of quantum electrodynamics (QED). The result takes the following covariant form,

$$
\begin{aligned}
\mathscr{L}= & \frac{f_{\pi}^{2}}{2}\left(\partial_{\mu} \phi\right)^{2}+m_{\pi}^{2} f_{\pi}^{2} \cos \phi+\frac{\mu}{8 \pi^{2}} \varepsilon^{0 \mu v \alpha} F_{\mu v} \partial_{\alpha} \phi-\frac{1}{32 \pi^{2}} \phi \varepsilon^{\mu v \alpha \beta} F_{\mu v} F_{\alpha \beta} \\
& -\frac{1}{4} F_{\mu v} F^{\mu v}-\frac{1}{2 \xi}\left(\partial_{\mu} A^{\mu}\right)^{2}-j_{\text {back }}^{\mu} A_{\mu},
\end{aligned}
$$

where $A_{\mu}$ is the gauge potential of QED, $F_{\mu \nu} \equiv \partial_{\mu} A_{\nu}-\partial_{\nu} A_{\mu}$ the corresponding field-strength tensor, and $\xi$ is the gauge-fixing parameter. Finally, $j_{\text {back }}^{\mu}$ represents a classical charged background, which is introduced in order to ensure local electric neutrality of the CSL ground state [4].

\subsection{Modification of the excitation spectrum}

In order to understand how the presence of dynamical electromagnetic fields affects the spectrum of excitations above the CSL ground state, the equations of motion will be utilized. Using the nonrelativistic notation, these take the following form in the Lorenz gauge, $\partial_{\mu} A^{\mu}=0$,

$$
\begin{aligned}
\ddot{\phi}-\nabla^{2} \phi+m_{\pi}^{2} \sin \phi & =\frac{1}{4 \pi^{2} f_{\pi}^{2}} \boldsymbol{E} \cdot \boldsymbol{B}, \\
\nabla \cdot \boldsymbol{E} & =\rho_{\text {back }}-\frac{1}{4 \pi^{2}} \boldsymbol{B} \cdot \boldsymbol{\nabla} \phi, \\
\boldsymbol{\nabla} \times \boldsymbol{B} & =\boldsymbol{j}_{\text {back }}+\dot{\boldsymbol{E}}+\frac{1}{4 \pi^{2}} \boldsymbol{B} \dot{\phi}-\frac{1}{4 \pi^{2}} \boldsymbol{E} \times \boldsymbol{\nabla} \phi .
\end{aligned}
$$

They can be further simplified by linearizing in small fluctuations around the CSL and magnetic field backgrounds, and subsequently Fourier-transforming in both time and the transverse coordinates. Upon the Fourier transform, the fluctuation of the magnetic field can be expressed in terms of the fluctuation of the electric field via the Bianchi identity $\boldsymbol{\nabla} \times \boldsymbol{E}=-\dot{\boldsymbol{B}}$. At the end of the day, one arrives at a set of four coupled linear differential equations for the neutral pion fluctuation $\pi$ and the electric field fluctuation $\boldsymbol{E}$,

$$
\begin{array}{r}
f_{\pi}\left(-\partial_{z}^{2}-\omega^{2}+\boldsymbol{p}_{\perp}^{2}+m_{\pi}^{2} \cos \phi\right) \pi-C B E_{z}=0, \\
\mathrm{i} f_{\pi} \omega^{2} C B \pi+\boldsymbol{p}_{\perp} \cdot \partial_{z} \boldsymbol{E}_{\perp}+\mathrm{i}\left(\omega^{2}-\boldsymbol{p}_{\perp}^{2}\right) E_{z}=0, \\
\mathrm{i}\left(\partial_{z}^{2}+\omega^{2}-p_{y}^{2}\right) E_{x}+\left(\mathrm{i} p_{x} p_{y}+\omega C f_{\pi} \partial_{z} \boldsymbol{\phi}\right) E_{y}+p_{x} \partial_{z} E_{z}=0, \\
\left(\mathrm{i} p_{x} p_{y}-\omega C f_{\pi} \partial_{z} \phi\right) E_{x}+\mathrm{i}\left(\partial_{z}^{2}+\omega^{2}-p_{x}^{2}\right) E_{y}+p_{y} \partial_{z} E_{z}=0 .
\end{array}
$$

Here $\phi$ and $\boldsymbol{B}$ represent the CSL condensate and the background magnetic field, respectively, and $C \equiv 1 /\left(4 \pi^{2} f_{\pi}\right)$ is a shorthand notation for the sake of brevity. 
In the chiral limit, the CSL background has a constant gradient, $f_{\pi} \partial_{z} \phi=\mu C B$, and one can thus Fourier transform in the $z$-coordinate as well. It is then straightforward to find the leading contributions to the dispersion relations of all the excitation branches in the low-energy limit,

$$
\begin{aligned}
& \omega_{1}=\frac{|\boldsymbol{p}|}{C B} \sqrt{\boldsymbol{p}_{\perp}^{2}+\frac{p_{z}^{2}}{\mu^{2} C^{2}}}+\mathscr{O}\left(\boldsymbol{p}^{4}\right), \\
& \omega_{2}=\mu C^{2} B+\mathscr{O}\left(\boldsymbol{p}^{2}\right), \\
& \omega_{3}=C B+\mathscr{O}\left(\boldsymbol{p}^{2}\right) .
\end{aligned}
$$

There are three different modes as expected, corresponding to (mixtures of) the neutral pion and two photon polarizations. Somewhat less expected is that two of the modes are gapped and only one is gapless, in spite of the spontaneously broken exact translation invariance and the gauge invariance protecting the photon degrees of freedom. The dispersion relation of the gapless mode is anisotropic, but quadratic in all directions. This is in contrast to the findings of ref. [9], based on an analysis of the propagation of electromagnetic fields on the fixed CSL background, where the low-energy dispersion relation is quadratic only in the direction of the magnetic field, but linear in the transverse directions. An explicit calculation of the matrix propagator shows that in spite of its soft low-energy behavior, the gapless mode gives rise to an infrared-finite two-point correlation function. This guarantees the absence of the Landau-Peierls instability [10,11], and the long-range order of the CSL phase is thus expected to survive up to some nonzero critical temperature.

Away from the chiral limit, the set of equations of motion (5.3) seems difficult to solve analytically, even in a series expansion in powers of momentum. What is easier to deal with is the case of modes propagating in the $z$-direction, that is along the magnetic field. In this case, the residual symmetry under rotations around the $z$-axis admits a well-defined notion of helicity, which in turns allows one to distinguish the pion and photon degrees of freedom: the set of equations (5.3) can be separated. The first pair of equations describes the propagation of the neutral pion, and reduces to

$$
\left(-\partial_{z}^{2}-\omega^{2}+C^{2} B^{2}+m_{\pi}^{2} \cos \phi\right) \pi=0,
$$

together with an explicit solution for the longitudinal electric field, $E_{z}=-f_{\pi} C B \pi$. This is a straightforward generalization of eq. (4.1), obtained by the replacement $\omega^{2} \rightarrow \omega^{2}-C^{2} B^{2}$ therein. Thus, all the results obtained in section 4.1 remain valid, except that the squared frequency of the neutral pion mode is shifted by $C^{2} B^{2}$. As a consequence of the coupling to the dynamical electromagnetic field, this mode is no longer gapless, but rather receives a gap equal to $B /\left(4 \pi^{2} f_{\pi}\right)$.

In the photon sector, the residual rotation invariance allows one to define helicity eigenstates, $E_{ \pm} \equiv E_{x} \pm \mathrm{i} E_{y}$. Their propagation is governed by the differential equation

$$
\left(-\partial_{z}^{2}-\omega^{2} \pm \omega C f_{\pi} \partial_{z} \phi\right) E_{ \pm}=0 .
$$

Despite its apparent simplicity, this equation is not easy to solve in a closed form. What one can say in general is that the presence of the term linear in frequency makes the dispersion relation of one of the circularly polarized photons quadratic, whereas the other polarization becomes gapped. In the long-wavelength limit, it is possible to obtain approximate analytic expressions for the dispersion relations [4],

$$
\omega_{+}=4 \pi k K(k) \frac{p_{z}^{2}}{m_{\pi}}+\mathscr{O}\left(p_{z}^{4}\right), \quad \omega_{-}=\frac{m_{\pi}}{4 \pi k K(k)}+\mathscr{O}\left(p_{z}^{2}\right)
$$




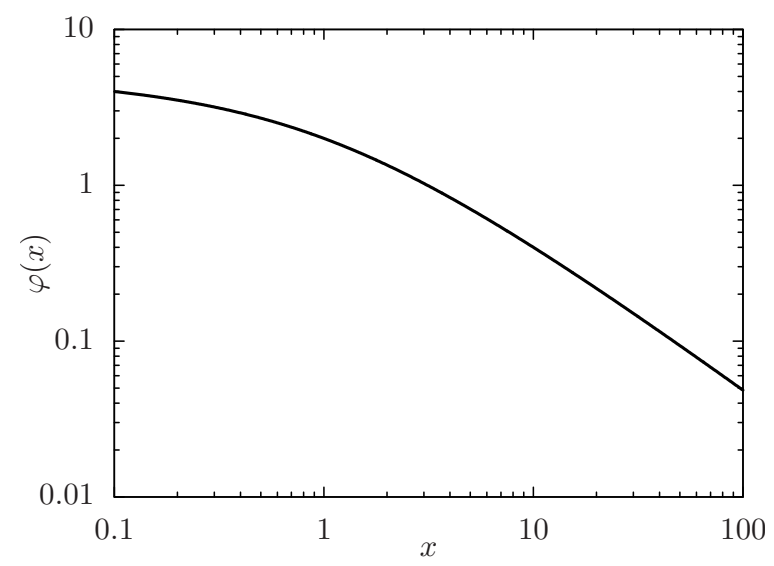

Figure 6: Anisotropy factor $\varphi(x)$, defined by eq. (5.10). Note the logarithmic scale on both axes. This figure is taken over from ref. [4].

\subsection{Consequences for QCD thermodynamics}

Understanding the spectrum of the system is determinant for its thermodynamic properties. At the leading order in the thermal loop expansion, the system can be treated as a gas of free quasiparticles with the above-calculated dispersion relations $\omega_{i}(\boldsymbol{p})$, see eq. (5.4). The thermal part of pressure is then given by

$$
P=-T \sum_{i=1}^{3} \int \frac{\mathrm{d}^{3} \boldsymbol{p}}{(2 \pi)^{3}} \log \left[1-e^{-\beta \omega_{i}(\boldsymbol{p})}\right] .
$$

Due to the complicated form of the dispersion relations, it does not seem possible to evaluate the thermal pressure in a closed form even in the chiral limit. However, it is still possible to get a simple, approximate expression for pressure, valid at sufficiently low temperatures. Namely, at temperatures $T \ll \min \left\{\mu C^{2} B, C B\right\}$, the contribution of the gapped modes is exponentially suppressed. At the same time, only the leading, quadratic part of the dispersion relation $\omega_{1}$ in eq. (5.4) contributes. This leads to an integral expression for the pressure that is possible to evaluate in a closed form,

$$
P=\frac{\zeta\left(\frac{5}{2}\right)}{16 \pi^{3 / 2}}(C B)^{3 / 2} T^{5 / 2} \varphi\left(\frac{1}{\mu C}\right)
$$

up to a dimensionless temperature-independent factor,

$$
\varphi(x) \equiv \int_{0}^{\pi} \frac{\sin \theta \mathrm{d} \theta}{\left(\sin ^{2} \theta+x^{2} \cos ^{2} \theta\right)^{3 / 4}} .
$$

The scaling of the pressure with temperature and magnetic field follows immediately from dimensional analysis and the form of the dispersion relation $\omega_{1}(\boldsymbol{p})$ in eq. (5.4). The chemical potential only affects the anisotropy thereof, which is why it only enters the pressure through the anisotropy factor $\varphi\left(\frac{1}{\mu C}\right)$. The numerical values of this factor are displayed in figure 6. For realistic values of the chemical potential, the parameter $1 /(\mu C)$ will always be larger than one, and accordingly $\varphi$ will take values of order one or smaller. 


\section{Conclusions and outlook}

In sufficiently strong magnetic fields and at moderate values of the baryon chemical potential, the ground state of QCD is a periodic lattice of topological solitons: the Chiral Soliton Lattice (CSL). The prediction of this new phase of dense matter is based on the low-energy EFT of QCD and is thus model-independent. Within the used truncation of the EFT action, which is controlled by the derivative expansion thereof, the results for the CSL ground state are exact and fully analytic. To the best of my knowledge, this is the first time that the existence of an inhomogeneous phase in the phase diagram of QCD has been demonstrated beyond doubt due to, for instance, the choice of model or approximation scheme.

The coupling of the CSL background to dynamical electromagnetic fields gives rise to a soft mode whose dispersion relation is anisotropic, yet quadratic in all directions in the low-energy limit. This mode gives a peculiar contribution to pressure, scaling with temperature and magnetic field as $T^{5 / 2} B^{3 / 2}$. At sufficiently low temperatures, this dominates over the usual black-body radiation pressure, and thus constitutes the leading, hitherto unnoticed, contribution to the pressure of QCD+QED. Note that even at zero baryon chemical potential, when the CSL state is absent, the chiral anomaly in presence of a background magnetic field still leads to a mixing of the neutral pion and photon degrees of freedom. This results in a modification of the excitation spectrum, and eventually in a contribution to thermal pressure that scales as $T^{3} B$ [12].

Out of possible extensions of the results presented here, let me mention one, which is currently under investigation in collaboration with Georgios Filios and Helena Kolešová. It is namely possible to demonstrate that an inhomogeneous phase similar to CSL exists also in the phase diagram of certain QCD-like theories that, unlike QCD itself, do not suffer from the sign problem. The simplest example of such a theory is QCD based on the SU(2) gauge group with two flavors of fundamental quarks, but the same conclusion can be made about the whole class of QCD-like theories with quarks in a real or pseudoreal representation of the gauge group. The presence of an inhomogeneous CSL-like phase in the phase diagram of such theories has already been established using EFT methods. Complete results regarding the detailed phase structure will be published elsewhere. This opens the way to investigation of inhomogeneous phases of matter using lattice Monte Carlo techniques.

\section{Acknowledgments}

I gratefully appreciate collaboration with Georgios Filios, Saurabh Kadam, Helena Kolešová and especially Naoki Yamamoto, on which this contribution is based. I would also like to thank the organizers of the parallel session "Nuclear and Astroparticle Physics" of the Confinement2018 conference for the invitation to give a talk within the session. Last but not least, I am indebted to the audience that attended my talk, in particular to Michael Buballa, Maxim Chernodub, Tom Cohen, Pawel Danielewicz, Eugenio Megías, Andreas Schmitt and Igor Shovkovy, for inspiring comments and stimulating subsequent discussions. The research reported here was supported in part by the grant no. PR-10614 within the ToppForsk-UiS program of the University of Stavanger and the University Fund. 


\section{References}

[1] J. O. Andersen, W. R. Naylor, and A. Tranberg, Phase diagram of QCD in a magnetic field: A review, Rev. Mod. Phys. 88 (2016) 025001, [arXiv:1411. 7176].

[2] V. A. Miransky and I. A. Shovkovy, Quantum field theory in a magnetic field: From quantum chromodynamics to graphene and Dirac semimetals, Phys. Rept. 576 (2015) 1-209, [arXiv:1503.00732].

[3] T. Brauner and N. Yamamoto, Chiral Soliton Lattice and Charged Pion Condensation in Strong Magnetic Fields, JHEP 04 (2017) 132, [arXiv: 1609 . 05213].

[4] T. Brauner and S. Kadam, Anomalous electrodynamics of neutral pion matter in strong magnetic fields, JHEP 03 (2017) 015, [arXiv: 1701 . 06793].

[5] D. T. Son and M. A. Stephanov, Axial anomaly and magnetism of nuclear and quark matter, Phys. Rev. D77 (2008) 014021, [arXiv: 0710.1084 ].

[6] J.-i. Kishine and A. S. Ovchinnikov, Theory of monoaxial chiral helimagnet, Solid State Physics 66 (2015) 1-130.

[7] F. Finkel, A. González-López, and M. A. Rodríguez, A new algebraization of the Lamé equation, J. Phys. A: Math. Gen. 33 (2000) 1519-1542.

[8] H. Li, D. Kusnezov, and F. Iachello, Group theoretical properties and band structure of the Lamé Hamiltonian, J. Phys. A: Math. Gen. 33 (2000) 6413-6429.

[9] N. Yamamoto, Axion electrodynamics and nonrelativistic photons in nuclear and quark matter, Phys. Rev. D93 (2016) 085036, [arXiv: 1512.05668].

[10] T.-G. Lee, E. Nakano, Y. Tsue, T. Tatsumi, and B. Friman, Landau-Peierls instability in a Fulde-Ferrell type inhomogeneous chiral condensed phase, Phys. Rev. D92 (2015), no. 3034024, [arXiv:1504.03185].

[11] Y. Hidaka, K. Kamikado, T. Kanazawa, and T. Noumi, Phonons, pions and quasi-long-range order in spatially modulated chiral condensates, Phys. Rev. D92 (2015), no. 3 034003, [arXiv:1505.00848].

[12] T. Brauner and S. V. Kadam, Anomalous low-temperature thermodynamics of QCD in strong magnetic fields, JHEP 11 (2017) 103, [arXiv: 1706.04514 ]. 